\title{
Optimized Trajectories of the Transfer Cask System in ITER
}

\author{
Filipe Valente $^{\mathrm{a}}$, Alberto Vale ${ }^{\mathrm{a}, *}$, Daniel Fonte ${ }^{\mathrm{a}}$, Isabel Ribeiro ${ }^{\mathrm{b}}$ \\ ${ }^{a}$ Instituto de Plasmas e Fusão Nuclear - Laboratório Associado, Instituto Superior Técnico, Av. Rovisco Pais 1, 1049-001 Lisboa - Portugal \\ ${ }^{b}$ Institute for Systems and Robotics, Instituto Superior Técnico, Av. Rovisco Pais 1, 1049-001 Lisboa - Portugal
}

\begin{abstract}
The Transfer Cask System(TCS) is one of the remote handling systems that will operate in ITER, transporting heavy and highly activated in-vessel components between the Tokamak Building and the Hot Cell Building. A motion planning methodology for the TCS was developed, providing smooth paths that maximize the clearance to obstacles and that incorporate manoeuvres whenever necessary. This paper presents the results of the TCS planning algorithm with trajectories computed for nominal operations. The length of the journey, the velocity, the time duration, and the risk of collision were evaluated individually for each trajectory. A summary of all results, conclusions and future work are presented and discussed.
\end{abstract}

Keywords: ITER, Remote Handling, Motion Planning, Transfer Cask System, Cask and Plug Remote Handling System

\section{Introduction}

The ITER (International Thermonuclear Experimental Reactor) is a joint international research project, aiming to demonstrate the technological feasibility of fusion power as an alternative and safe power source. During maintenance operations human presence will not be allowed in ITER's Tokamak Building (TB) and Hot Cell Building (HCB). Therefore, the setup of an effective ITER Remote Maintenance System (IRMS) is of vital importance to the project.

The Transfer Cask System (TCS), also known as Cask and Plug Remote Handling System (CPRHS), is the transport platform operating between the TB and the HCB, as shown in Figure 1. There are up to 8 different TCS configurations, each defined according to the required activity. The largest TCS has dimensions $8.5 \mathrm{~m} \times 2.62 \mathrm{~m} \times 3.62 \mathrm{~m}$ (length, width, height) and is entrusted with the transportation of heavy (up to 45T) and highly activated (gamma dose rates in excess of $100 \mathrm{~Gy} / \mathrm{h}$ ) components [1]. It comprises three sub-systems: a cask envelope containing the load, a pallet that supports the cask envelope, and the Air Transfer System (ATS). The ATS drives the entire TCS by means of an air-cushion system. Its kinematic configuration, first proposed in [2], endows it with the required flexibility to navigate autonomously or remotely controlled, in the cluttered environments of the TB and the HCB. During the reactor's operation, the in-vessel components, such as the blankets that cover the vacuum vessel, are expected to become activated by neutron exposure. When such components have to be removed for disposal, operations are to be carried out by the TCS, which is required to dock in pre-defined locations, the vacuum vessel port cells (VVPC), located on the three levels of TB: B1 (divertor level), L1 (equatorial level) and L2 (upper level). Due to

${ }^{*}$ Corresponding author. Tel.:+351 218419 098; fax:+351 218417819.
Email address: avale@ipfn.ist.utl.pt (Alberto Vale)

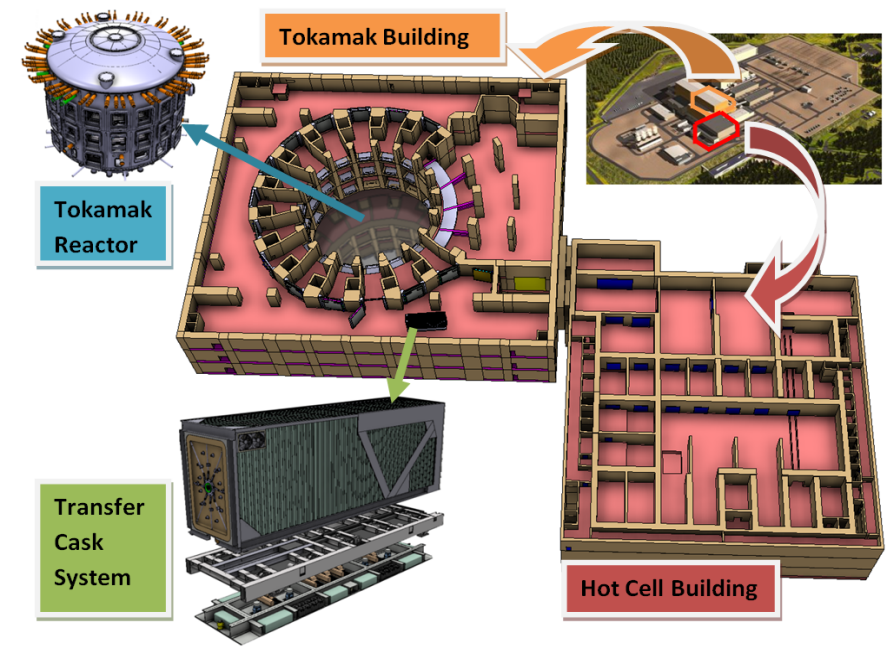

Figure 1: The CAD models of TB and HCB scenarios. Also displayed are detail views of the TCS, the reactor and the ITER site.

the confined environment, manoeuvres play an important role for entering/exiting the VVPCs and the lift connecting the TB and the HCB. Operations in the HCB include the diagnose and refurbishment or disposal of activated components. Hence, the TCS must dock at the docking stations through a Port Plug (PP) interface or park in the Parking Areas (PA) at different levels of the $\mathrm{HCB}$, in this paper referred as B2, B1, L1 and L3.

The TCS trajectories must be optimized in order to maximise the distance to the obstacles and the motion smoothness, while minimising the path length. Different approaches were studied and a motion planning framework was developed [3]. In this paper the main results of the study are presented and discussed.

This paper is organized as follows: Section 2 describes the motion planning methodology, Section 3 presents the results, followed by the conclusions and future work in Section 4. 


\section{Motion Planning Methodology}

\subsection{Optimal Trajectories}

For a TCS safe motion, a path maximising the distance to obstacles and minimizing the distance between the start and the goal poses (position and orientation) must be evaluated. By specifying the TCS velocity along the path, the optimized trajectory is obtained. To meet these conditions a motion planning methodology was developed and implemented, [3]. It is achieved in four main steps, as displayed in Figure 2: 1) generation of a 2D map of the environment from its 3D model, 2) evaluation of a geometric path, 3) path optimization, and 4) building a trajectory from the optimized path. A description of each follows.

Step 1 - From 3D CAD models, a 2D representation is obtained by projection at floor level, including all the relevant elements that might conflict with the TCS volume. The TB and the HCB are well structured scenarios that can be modelled as a set of planar walls, whose footprint is a line segment and thus the 2D map can be considered as a set of line segments.
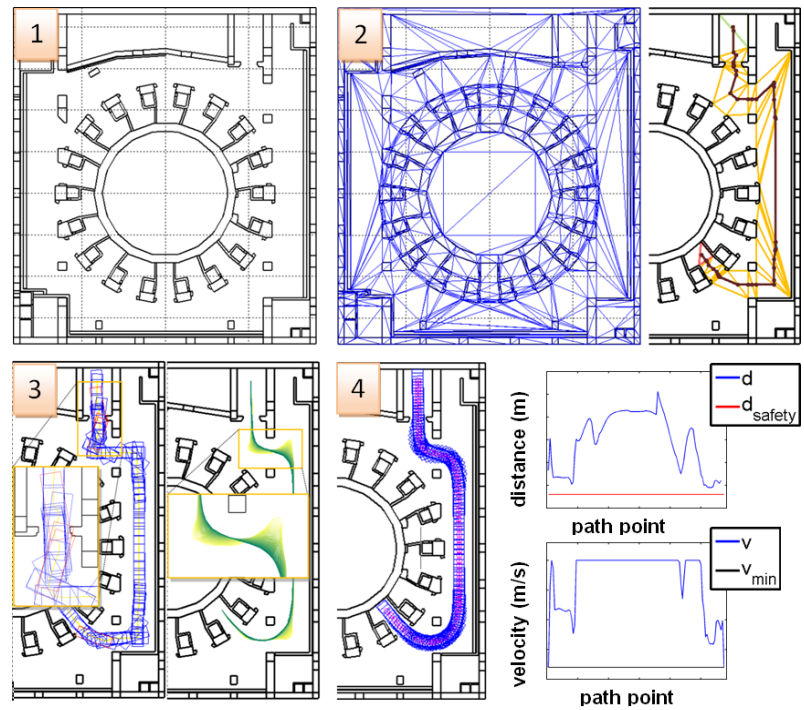

Figure 2: Motion planning methodology. Step 1: generation of the 2D map of the environment. Step 2: evaluation of a geometric path. Step 3: path optimization. Step 4: final optimized trajectory, with the evolution of the distance to obstacles and velocity along the path.

Step 2 - Find a collision free geometric path, i.e., a set of 2D points that connects the start and goal points and do not intersect the line segments of the map. For that, the 2D map is decomposed into a set of triangles, a typical cell decomposition [5], [6], by using Constrained Delaunay Triangulation (CDT), [4], to account for all walls. Afterwards the algorithm determines all sets of sequences of triangles that contain and link the start and goal points. Each sequence of triangles is then converted into a sequence of points (mid point of the common edge of two consecutive triangles) yielding a path. The shortest and feasible path is chosen as the geometric path (step 2 of Figure 2), acting as the initial condition for the path optimization module.

Step 3 - The so obtained geometric path does not guarantee a collision free path for a rigid body, such as the TCS, as illustrated in the left image of step 3 of Figure 2 with a TCS collision in red, and thus may be unfeasible. Moreover this path is not smooth. To obtain an optimized path, two criteria are included in the algorithm: clearance from obstacles, by increasing the distance from the TCS to walls, and path smoothness, entailing getting shorter and smoother paths without slacks. To address the referred issues, the optimization procedure uses the elastic band concept, [7], where the path is modelled as an elastic band, similar to a series of connected springs, subjected to two types of forces: internal and external forces. The first are the internal contraction forces, whose magnitude is proportional to the amplitude of displacement and determine that the paths becomes retracted and shorter. The repulsive forces are responsible for keeping the path, and consequently the vehicle, away from obstacles.

Step 4 - To define the velocity of the TCS along each point of the optimized path, the TCS velocity, $v$, is defined as a function of the distance to the nearest obstacle, $d$. When $d$ is above a given threshold, $d_{\text {threshold }}$, a maximum allowable velocity for the TCS, $v_{\max }$, is assumed. To avoid the situation where the motors' torque is not sufficient to overcome friction, a minimum allowable velocity, $v_{\min }$, is considered for distances smaller than the minimum safety distance, $d_{\text {safety. }}$ Otherwise, when $d_{\text {safety }} \leq d \leq d_{\text {threshold }}$ the velocity varies linearly between $v_{\min }$ and $v_{\max }$. To be compliant with ITER building tolerances, TCS manufacturing and assembly tolerances and TCS positional inaccuracies, $d_{\text {safety }}=0.3 \mathrm{~m}$, and $d_{\text {threshold }}=1 \mathrm{~m}$. The following values were also adopted: $v_{\min }=0.02 \mathrm{~m} / \mathrm{s}$ and $v_{\text {max }}=0.2 \mathrm{~m} / \mathrm{s}$ for all nominal operations.

\subsection{Manoeuvres}

There are particular situations where the described methodology fails to generate feasible solutions, due to the confined environment. The inclusion of manoeuvres can greatly improve the path planning, by providing a feasible solution where none could be found before, but also by improving the distance to obstacles. A manoeuvre exists when the TCS stops and changes its motion direction, so as to achieve a specified orientation. A manoeuvre requires splitting the path in two sub-paths with the constraint that the final pose of the first sub-path is the initial pose of the next sub-path. By taking advantage of the TCS
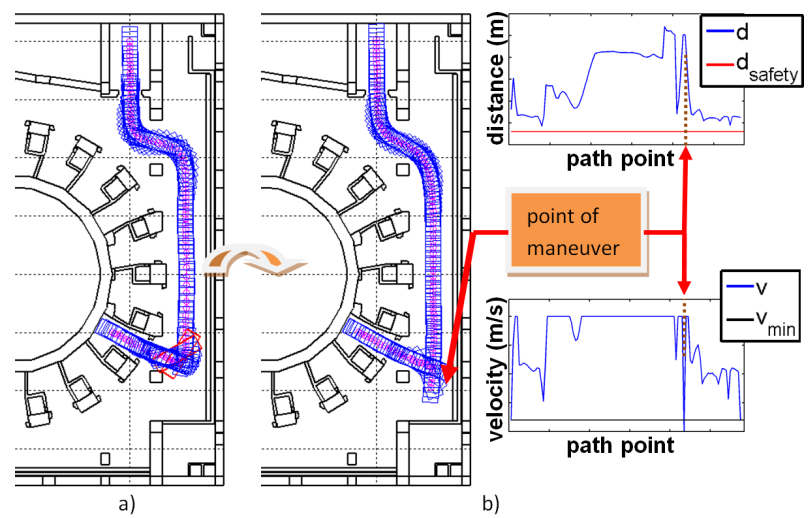

Figure 3: Example for VVPC 17 in level B1 of TB: a) trajectory without maneuver, with collision (red); b) optimized trajectory with maneuver, minimum distance to obstacles and velocity along the path. 
kinematic configuration, the algorithm in [3], was improved to incorporate one or multiple manoeuvres. In case $n$ manoeuvres are required, the path is divided in $n+1$ sub-paths and the path optimization is applied to each. The decision of including manoeuvres is taken when a path without manoeuvre is not feasible, as illustrated in Figure 3 a) with a collision in red, or does not fulfil the minimum safety distance to obstacles. The algorithm requires for the point(s) of manoeuvre to be introduced manually, adjusting its position(s) during the optimization to obtain the final trajectory, as displayed in Figure $3 \mathrm{~b}$ ).

\section{Results}

The optimized trajectories were computed assuming a navigation strategy where both wheels of the ATS follow the same path. The trajectories presented in this section consider that the initial pose is inside the lift and the final pose is at the destination (VVPC, PP or PA) being valid for both directions. However, trajectories can be generated between any two poses.

For each operation a start and goal poses were introduced and, if required, additional manoeuvre point(s) were supplied. For each level statistic results are presented focusing on the number of manoeuvres (M), length (L) and duration (D) of journey. The duration did not take into account the period of time the ATS requires to be stopped (e.g., while a VVPC door is opening). This allows to set a minimum boundary reference for the time to complete each nominal operation. The average distance to obstacles for all trajectories on each level is also displayed.

A total of 46 trajectories were computed on TB from which 18 have at least one manoeuvre (14 with one manoeuvre and 4 with two manoeuvres). The set of all trajectories on all three levels is shown in Figure 4. For each trajectory, the distance to

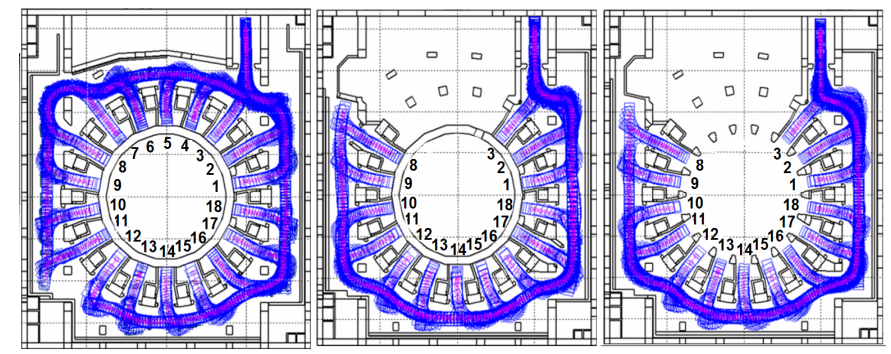

Figure 4: Set of all trajectories in TB: (from left to right) levels B1, L1 and L2.

the closest obstacle along the TCS motion was calculated, together with a proposed velocity profile. The example of VVPC 17 in level L1 is shown in Figure 5, where the walls were extended given that two TCSs (main IVT cask and intermediate IVT cask) will operate on it to support the Blanket RH System. The area spanned by the TCS, together with the evolutions of the distance to the closest obstacle and the velocity along the path are also shown. Clearly, the VVPC entrance period presents the higher risk of collision. The main statistical results for TB are presented in Table 1. The longest journey is to VVPC 9 in level L1 of TB taking approximately 20 minutes to complete $183.6 \mathrm{~m}$. The shortest journey is on level B1
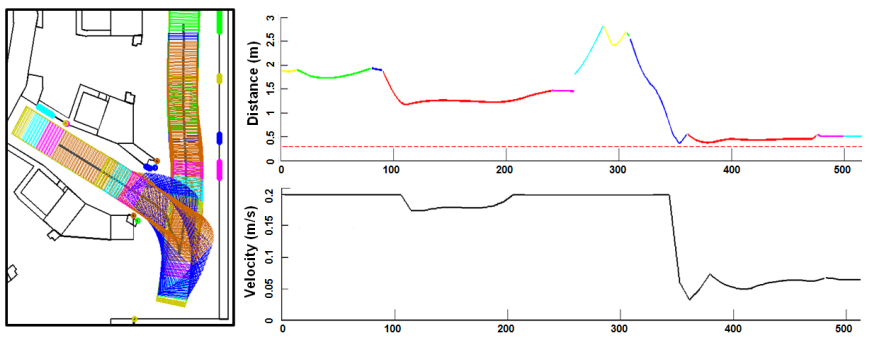

Figure 5: TCS entering in VV port cell 17 in Level L1 of TB. Left: area spanned along the path; Right: distance to the closest obstacle (with a color correspondence with spanned area on the left) and velocity profile.

\begin{tabular}{|c|ccc|ccc|ccc|}
\hline Level & \multicolumn{3}{|c|}{ B1 } & & \multicolumn{3}{c|}{ L1 } & \multicolumn{3}{c|}{ L2 } \\
\hline \hline VVPC & M & L & D & M & L & D & M & L & D \\
& & $(\mathrm{m})$ & $(\mathrm{s})$ & & $(\mathrm{m})$ & $(\mathrm{s})$ & & $(\mathrm{m})$ & $(\mathrm{s})$ \\
\hline \hline 1 & 0 & 51.9 & 465 & 0 & 51.2 & 471 & 0 & 51.2 & 473 \\
2 & 0 & 44.9 & 351 & 0 & 44.2 & 396 & 0 & 44.2 & 403 \\
3 & 0 & 29.8 & 290 & 0 & 30.4 & 397 & 0 & 30.4 & 400 \\
4 & 1 & 51.6 & 433 & & & & & & \\
5 & 1 & 63.2 & 394 & & & & & & \\
6 & 2 & 91.3 & 688 & & & & & & \\
7 & 2 & 97.6 & 762 & & & & & & \\
8 & 1 & 104.2 & 633 & 1 & 185.5 & 1176 & 1 & 185.9 & 1112 \\
9 & 1 & 109.8 & 770 & 1 & 183.6 & 1182 & 1 & 183.6 & 1182 \\
10 & 2 & 136.5 & 832 & 0 & 152.3 & 996 & 0 & 151.8 & 960 \\
11 & 2 & 143.3 & 850 & 0 & 145.6 & 992 & 0 & 145.9 & 915 \\
12 & 0 & 131.6 & 833 & 0 & 130.3 & 915 & 0 & 130.3 & 894 \\
13 & 0 & 118.8 & 813 & 0 & 118.4 & 854 & 0 & 118.4 & 838 \\
14 & 0 & 107.3 & 818 & 0 & 105.9 & 833 & 0 & 105.9 & 817 \\
15 & 0 & 96.6 & 708 & 0 & 97.4 & 739 & 0 & 97.4 & 742 \\
16 & 0 & 86.1 & 614 & 0 & 86.5 & 677 & 0 & 86.0 & 654 \\
17 & 1 & 83.7 & 546 & 1 & 88.2 & 633 & 1 & 83.9 & 567 \\
18 & 1 & 77.2 & 560 & 1 & 77.1 & 585 & 1 & 77.1 & 585 \\
\hline Totals & 14 & $1.6 \mathrm{e} 3$ & $11 \mathrm{e} 3$ & 4 & $1.5 \mathrm{e} 3$ & $11 \mathrm{e} 3$ & 4 & $1.5 \mathrm{e} 3$ & $11 \mathrm{e} 3$ \\
\hline
\end{tabular}

Table 1: Main results for all trajectories in TB, where: $M$ is the number of maneuvers, $\mathrm{L}$ the length of journey and $\mathrm{D}$ the duration of journey.

to VVPC 3 taking 4.8 minutes to travel $30 \mathrm{~m}$. The VVPC 14 trajectories, on all levels, are the most critical, since it was not possible to achieve a minimum distance above $d_{\text {safety }}$ along the whole trajectory (a value $d=0.29 \mathrm{~m}$ was obtained in the VVPC entrance) and is the reason why in Figure 6 there is an average of $0.01 \%$ for $d<0.3 \mathrm{~m}$. Such situation is due to the lack of available space at the entrance of the VVPC, resulting from the existence of cables on the walls.

A total of 19 trajectories were computed for the four levels of HCB of which 4 have one manoeuvre. There are 6 parking trajectories in total. The set of all trajectories, between the lift and each docking (PP) or parking (PA) location, on all four levels, is shown in Figure 7 where each location is identified. In level L1, besides the lift, the TCS can also enter in the HCB from an alternative route, the Neutral Beam (NB), Figure 7 in c). Each trajectory was computed for the worst case scenario, i.e., whenever there is a parked or docked TCS in its close vicinity. The 

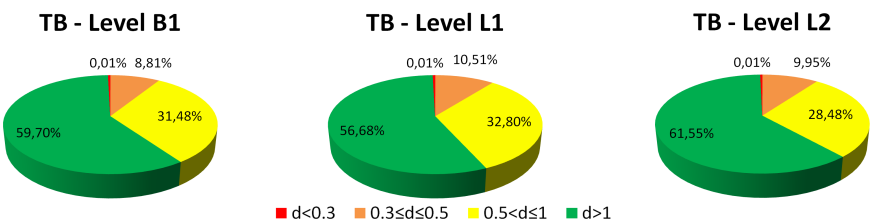

Figure 6: Average distance to obstacles for all trajectories, on each level of TB.

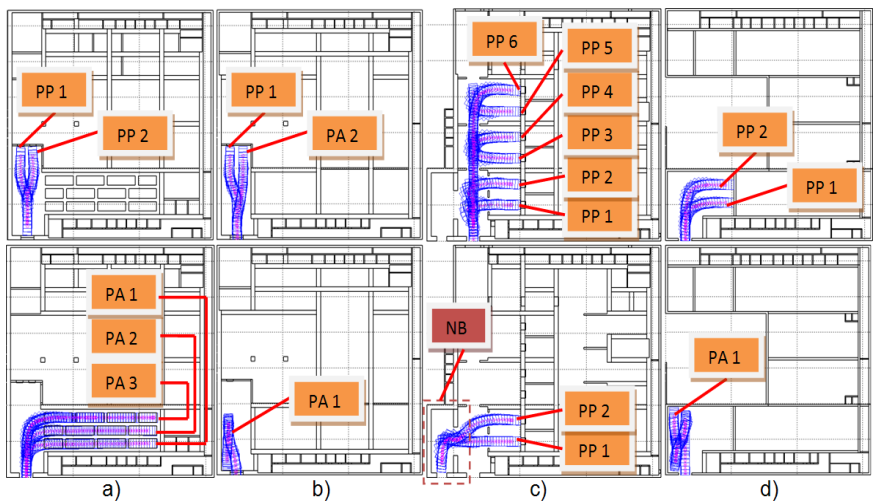

Figure 7: Set of all trajectories in HCB. In a): level B2; b): level B1; c): level L1; d): level L3. Top: docking locations; Bottom: parking locations

longest journey is to PP 5 on level L1 of HCB, taking 7 minutes in a trajectory of $53 \mathrm{~m}$. The shortest path is on level L3 to PP 1, taking 1.8 minutes to complete $20 \mathrm{~m}$, as indicated in Table 2. The most critical trajectory is to PA 1 in level B1, where the minimum distance goes below $d_{\text {safety }}$ (a $d=0.24 \mathrm{~m}$ was computed) this corresponding to the $4.72 \%$ value in Figure 8 . This situation occurs due to the low distance to the wall when the TCS is approaching the parking location.

\section{Conclusions and Future Work}

This paper presented the results of the TCS motion planning methodology in ITER scenario. Feasible trajectories were successfully computed for all TCS nominal operations and only in some particular situations it was not possible to achieve a $d>d_{\text {safety }}$, along the whole trajectory. However, the obtained minimum values are very close to $d_{\text {safety }}$. In fact, on average, $d$ is above $1 \mathrm{~m}$ in the TB and above $0.5 \mathrm{~m}$ in the HCB, which leads us to conclude that the latter is a more critical scenario when it comes to the available space for nominal operations. The inclusion of manoeuvres also proved to be a valuable improvement for the successful computation of feasible and safe trajectories. The trajectories were included in a CAD visualization tool, [8].

Future improvements will focus on path following strategies in a situation where both TCS wheels are not constrained to follow the same path which requires the integration of localization techniques as the one presented in [9]. Moreover, particular situations such as driving the ATS from beneath the pallet, the motion to implement parking logistics, and those involved in rescue and recover situations will be addressed.

\section{Acknowledgments}

Work supported by the grant F4E-GRT-016(MS-RH) funded by the European Joint Undertaking for ITER and the Develop-

\begin{tabular}{|c|ccc|ccc|ccc|ccc|}
\hline Level & \multicolumn{3}{|c|}{ B2 } & \multicolumn{3}{|c|}{ B1 } & & L1 & \multicolumn{3}{|c|}{ L3 } \\
\hline Location & M & $\begin{array}{c}\text { L } \\
(\mathrm{m})\end{array}$ & $\begin{array}{c}\text { D } \\
(\mathrm{s})\end{array}$ & M & $\begin{array}{c}\text { L } \\
(\mathrm{m})\end{array}$ & $\begin{array}{c}\mathrm{D} \\
(\mathrm{s})\end{array}$ & $\mathrm{M}$ & $\begin{array}{c}\mathrm{L} \\
(\mathrm{m})\end{array}$ & $\begin{array}{c}\mathrm{D} \\
(\mathrm{s})\end{array}$ & $\mathrm{M}$ & $\begin{array}{c}\mathrm{L} \\
(\mathrm{m})\end{array}$ & $\begin{array}{c}\text { D } \\
(\mathrm{s})\end{array}$ \\
\hline \hline PP 1 & 0 & 20.0 & 137 & 0 & 24.3 & 189 & 0 & 22.8 & 152 & 0 & 19.9 & 108 \\
PP 2 & 0 & 20.0 & 136 & & & & 0 & 23.9 & 188 & 0 & 25.0 & 175 \\
PP 3 & & & & & & & 1 & 39.6 & 256 & & & \\
PP 4 & & & & & & & 0 & 34.6 & 245 & & & \\
PP 5 & & & & & & & 1 & 52.9 & 395 & & & \\
PP 6 & & & & & & & 0 & 46.8 & 378 & & & \\
NB-PP 1 & & & & & & & 0 & 26.5 & 195 & & & \\
NB-PP 2 & & & & & & & 0 & 28.1 & 307 & & & \\
PA 1 & 0 & 44.0 & 306 & 1 & 25.5 & 365 & & & & 1 & 40.7 & 289 \\
PA 2 & 0 & 47.0 & 309 & 0 & 23.7 & 254 & & & & & & \\
PA 3 & 0 & 50.6 & 308 & & & & & & & & & \\
\hline Totals & 0 & 182 & $1 \mathrm{e} 3$ & 1 & 74 & 808 & 2 & 275 & $2 \mathrm{e} 3$ & 1 & 86 & 572 \\
\hline
\end{tabular}

Table 2: Main results for all trajectories in $\mathrm{HCB}$, where: $\mathrm{M}$ is the number of maneuvers, $\mathrm{L}$ the length of journey and $\mathrm{D}$ the duration of journey.

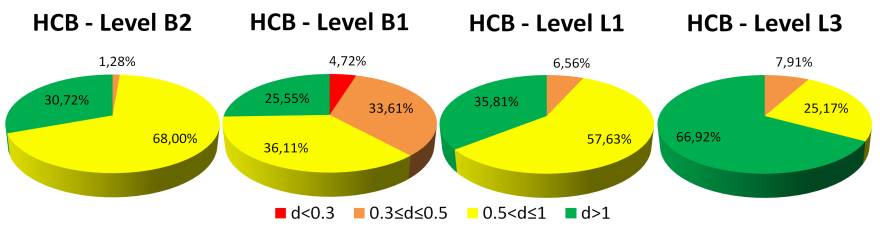

Figure 8: Average minimum distance to obstacles for all trajectories, on each level of $\mathrm{HCB}$.

ment of Fusion Energy (F4E-Fusion for Energy). The views expressed in this publication are the sole responsibility of the authors. F4E is not liable for the use which might be made of the information in this publication.

\section{References}

[1] C. Gutiérrez, C. Damiani, M. Irving, J-P. Friconneau, A. Tesini, M.I Ribeiro and A. Vale, "ITER Transfer Cask System: status of design, issues and future developments", Proceedings of the 9th International Symposium on Fusion Nuclear Energy, China (2009).

[2] M.I. Ribeiro, P. Lima, R. Aparício and R. Ferreira,"Conceptual Study on Flexible Guidance and Navigation for ITER Remote Handling Transport Casks", Proceedings of the 17th IEEE/NPSS Symposium on Fusion Engineering, pp. 969-972, San Diego, USA (1997).

[3] D. Fonte, F. Valente, A. Vale and M.I. Ribeiro,"A Motion Planning Methodology for Rhombic-like Vehicles for ITER Remote Handling Operations", Proceedings of the 7th IFAC Symposium on Intelligent Autonomous Vehicles, Lecce, Italy (2010).

[4] L.P. Chew, "Constrained Delaunay Triangulations", Proceedings of the Third Annual Symposium on Computational Geometry, pp. 215-222, Waterloo, Ontario, Canada (1987).

[5] J-C. Latombe, "Robot Motion Planning", Kluwer Academic Publishers, Boston, MA (1991).

[6] S.M. La Valle,"Planning Algorithms", Cambridge University Press (2006).

[7] S. Quinlan and O. Khatib," Elastic Bands: Connecting Path Planning and Control", Proceedings IEEE Conference Robotics and Automation, vol. 2, pp. 802-807, Atlanta, USA(1993).

[8] P. Ruibanys, C. Reig, E. Gazeau, J. Marmie and N. Etchegoin, "Definition, Development and Operation of a Comprehensive Virtual Model of the ITER Buildings, ATS and CPRHS”, 26th Symposium on Fusion Technology, Porto (2010).

[9] J. Ferreira, A. Vale and R. Ventura, “ Optimizing Range Finder Sensor Network Coverage in Indoor Environment", Proceedings of the 7th IFAC Symposium on Intelligent Autonomous Vehicles, Lecce, Italy (2010). 